\section{Assessment of spatial-contrast function and short-wavelength sensitivity deficits in patients with migraine}

Ö Yenice', S Onal'1', B Incili', A Temel', N Afşar² and $T$ Tanrıdağ
${ }^{1}$ Marmara University School of Medicine, Department of Ophthalmology, Istanbul, Turkey

${ }^{2}$ Marmara University School of Medicine, Department of Neurology, Istanbul, Turkey

Correspondence: Ö Yenice, Avcilar cad, Eser Apt, 27/10, Idealtepe, Istanbul, Turkey Tel/Fax: + 902163279538 E-mail: yeniceozlem@ yahoo.com

Received: 27 May 2005 Accepted in revised form: 29 November 2005

Published online: 3 February 2006

\section{Abstract}

Aims To study spatial-contrast function and short-wavelength sensitivity deficits in a migraine population with a disease duration of 30 years or less.

Materials and methods In this prospective, cross-sectional study, we evaluated 28 subjects with migraine headache and 15 nonheadache healthy controls. Visual fields were evaluated using the Humphrey Field Analyzer 750i and the $30-2$, blue and yellow threshold programme. Contrast sensitivity (CS) was measured at 1.5, 3, 6,12 , and $18 \mathrm{cpd}$ spatial frequencies, using the Functional Acuity Contrast Test (F.A.C.T. ${ }^{\mathrm{TM}}$ ). The results of the visual field parameters (mean defect (MD) and pattern standard defect (PSD)) and CS were compared with 15 age-equivalent normal subjects.

Results Short-wavelength amplitude perimetry (SWAP) parameters and CS scores at all spatial frequencies were significantly altered in the migraine patients when compared with the control subjects. Visual field parameters correlated significantly with contrast sensitivity scores: positively for $\mathrm{MD}$ ( $r=0.39, P=0.01 ; r=0.43, P=0.005 ; r=0.56$, $P=0.0001 ; r=0.45, P=0.003 ; r=0.48$, $P=0.0001)$ and negatively for PSD $(r=-0.45$, $P=0.003 ; r=-0.45, P=0.003 ; r=-0.51$, $P=0.001 ; r=-0.53, P=0.0001 ;$ and $r=-0.67$, $P=0.0001)$ at all $(1.5,3,6,12$, and $18 \mathrm{cpd})$ spatial frequencies, respectively. Migraine duration correlated negatively with MD $(r=-0.42$, $P=0.04)$ and positively with PSD $(r=0.42$, $P=0.03)$.

Conclusion Migraineurs had significantly altered visual field and contrast function at all spatial frequencies to the normal population.
These defects share some features with early stages of glaucoma and may relate a possibility for a common vascular disease pathogenesis in these two conditions. Eye (2007) 21, 218-223. doi:10.1038/sj.eye.6702251; published online 3 February 2006

Keywords: SWAP; contrast sensitivity; migraine; glaucoma

\section{Introduction}

Visual field deficits have been identified in migraineurs between migraine episodes. ${ }^{1-5}$ This abnormal visual field function does not seem to result from visual aura as deficits have also been reported in patients who do not experience visual aura. ${ }^{6-8}$ Although homonymous visual field deficits characteristic of cortical involvement have been reported after migraine, most are nonhomonymous and often are unilateral implying precortical involvement. ${ }^{1-3}$ Previously, contrast sensitivity deficits have also been reported in migraineurs with a history of disease duration for 30 years (median 7 ) or more. ${ }^{9}$ Untill now, there have been no reports in the literature studying the visual field defects and contrast processing in the same migraine population. In this study, we tried to assess the presence and correlation of deficits of shortwavelength sensitivity and spatial-contrast function on the same migraineurs with a disease duration of 30 years or less.

\section{Materials and methods}

Our study included 28 subjects who had migraine headache (aged 32-69 years) and 15 
nonheadache healthy controls (aged 22-53 years). All patients were examined and diagnosed as migraine, which met the criteria of International Headache Society. ${ }^{10}$ Informed consent was obtained from each subject after the nature of study was explained. Both tests, including visual field testing and contrast sensitivity function were determined with best corrected visual acuity by using the refractive set that was originally given by the Humphrey Field Analyzer II.

All patients had best corrected visual acuity of 20/20 and had a normal ocular health as assessed by slit-lamp, fundus examination, and tonometry $(<21 \mathrm{~mm} \mathrm{Hg})$. All had symmetrically cupped optic discs $(<0.3)$ without any abnormality (peripapillary haemorrhage, notch or focal thinning) of the neuroretinal rim.

The control group consisted of 15 subjects, free of headache (established by clinical interview), who were randomly selected from patients that came to our outpatient clinic for refraction problems. None of the encountered subjects had visual field testing before, and none had any known risk factors (history of glaucoma in the first degree relative, history of smoking, systemic hypertension or hypotension, diabetes, and increased sensitivity of fingertips, and limps in cold) for glaucoma suspect.

Control and migraine subjects underwent visual field testing twice at least 1 week apart to minimise learning effect. All visual fields with any abnormal reliability parameter (fixation losses $>33 \%$, false-positive responses $>33 \%$, or false-negative responses $>33 \%$ ) were excluded from the study. Migraine patients required having at least 1 week after a migraine attack to minimize possible transient effects on performance due to nausea or postmigraine fatigue. We performed ShortWavelength Automated Perimetry (SWAP), using Humphrey Field Analyzer II (model 750) with a Goldmann size V blue target (Omega 440-nm interference filter) projected on a $100 \mathrm{~cd} / \mathrm{m}^{2}$ yellow background.

Spatial-contrast sensitivity was assessed using the Functional Acuity Contrast Test (F.A.C.T. ${ }^{\mathrm{TM}}$ ) (Sterero Optical Co., Inc.) at $3 \mathrm{~m}$ distance and under normal office lighting. Lighting conditions were standardized with a lightmeter (Sterero Optical Co., Inc.) which is included with the F.A.C.T. test to insure the test accuracy. Lightmeter is holded $5 \mathrm{~cm}$ from the centre of the chart and the luminance of $85 \mathrm{~cd} / \mathrm{m}^{2}$ is measured, which was also indicated by the green area on the lightmeter. The F.A.C.T. ${ }^{\mathrm{TM}}$ chart consists of 45 sine-wave gratings arranged in five rows and nine columns. Sine-wave grating size changes in steps equal to one octave between the first four rows and half octave between the fourth and fifth row. The corresponding spatial frequencies are $1.5,3,6,12$, and 18 cycles per degree (cpd). The contrast step between each grating patch is $0.15 \log$ units. There is $50 \%$ loss or $100 \%$ gain in contrast for any two contrast step increase or decrease, respectively. The grating patch size is $1.7^{\circ}$ and exceeds the size of the macula. Gratings are tilted $+15^{\circ}, 0^{\circ},-15^{\circ}$ to keep them within the orientation bandwidth of visual channels. The patients were shown the test rows in a random sequence and were tested three times. Each response was recorded. A final contrast sensitivity score was determined by the lowest contrast patch having at least two of three correct responses.

Group comparisons were performed for mean deviation (MD) and the pattern standard deviation (PSD) of visual field testing by using nonparametric (MannWhitney $U$ ) tests. As the two eyes of each patient cannot be considered to be independent, the mean of right, and left, eye data of each patient was used in statistical analysis. Spearman correlations were performed between duration of migraine and visual field parameters, duration of migraine and contrast sensitivity scores, and visual field indices and contrast sensitivity scores. For statistical analysis, SPSS/PC 10.0 (SPSS Inc., Chicago, IL, USA) was used. A $P$-value $<0.05$ was considered as statistically significant.

\section{Results}

Migraine group consisted of two male and 13 female subjects (age: $33 \pm 11.8$ years). The control group included five male and 13 female subjects (age: $31.4 \pm 8.5$ years). The duration of disease was $8.96 \pm 7.75$ (1-30) years in the migraine group. All migraine and control subjects had intraocular pressures within normal range. There was no statistically significant difference between migraine and control groups with respect to age $(P=0.8)$, refractive error $(P=0.07)$, and intraocular pressure $(P=0.6)$. Table 1 presents clinical characteristics of both groups.

Nonparametric comparisons yielded statistically significant difference for MD $(P=0.04)$ and PSD $(P=0.002)$ values, being worse in migraine subjects. Table 2 presents visual field indices and reliability parameters of both groups and Figure $1 \mathrm{a}$ and $\mathrm{b}$ show the distribution of global indices of each participitant. Migraine patients had statistically significant lower mean contrast sensitivities when compared to control subjects (Table 3), and visual field parameters correlated significantly with contrast-sensitivity scores positively for MD and negatively for PSD at all spatial frequencies (Table 4). Figure 2 shows the distribution of mean contrast sensitivity values of each participitant.

When we studied the correlation between the duration of migraine and visual field parameters, a negative correlation was found for MD $(r=-0.42, P=0.04)$ and a positive correlation was found for PSD $(r=0.42$, 
Table 1 Clinical characteristics of migraine and control groups

\begin{tabular}{lccc}
\hline & Control $(\mathrm{n}=14)$ & Migraine $(\mathrm{n}=28)$ & $\mathrm{P}$ \\
\hline Age & $31.4 \pm 8.5$ & $33 \pm 11.8$ & 0.8 \\
Sex & & & \\
$\quad$ Male & 2 & 5 & \\
Female & 12 & 23 & \\
IOP (mmHg) & $12.9 \pm 0.7$ & $12.4 \pm 1.91$ & 0.6 \\
Refraction (SE) & $-0.87 \pm 1.61$ & $0.23 \pm 1.41$ & 0.07 \\
\hline
\end{tabular}

${ }^{*} \mathrm{SE}=$ spheric equivalent.

Table 2 Test time, reliability indices, and visual field parameters of migraine and control subjects

\begin{tabular}{lrrl}
\hline & Control & Migraine & \multicolumn{1}{c}{$\mathrm{P}^{\mathrm{a}}$} \\
\hline Visual field parameters & & & \\
MD & $5.23 \pm 3.13$ & $-7.91 \pm 4.19$ & $0.04^{*}$ \\
PSD & $3.03 \pm 0.39$ & $3.95 \pm 1.07$ & $0.002^{*}$ \\
Test time (min) & $15.0 \pm 2.01$ & $16.1 \pm 1.78$ & 0.8 \\
& & & \\
Reliability indices & & & \\
FN (\%) & $8.06 \pm 8.37$ & $7.06 \pm 8.01$ & 0.14 \\
FP (\%) & $1.95 \pm 4.48$ & $1.07 \pm 3.02$ & 0.17 \\
FL (\%) & $5.46 \pm 8.09$ & $6.09 \pm 7.12$ & 0.12 \\
\hline
\end{tabular}

$\mathrm{FN}=$ false-negative responses, $\mathrm{FP}=$ false-positive responses, $\mathrm{FL}=$ fixation losses.

${ }^{*} P<0.05$ indicates statistically significance.

${ }^{a}$ Mann-Whitney $U$ Test.

$P=0.03$ ), and the results were statistically significant. Figures 3 and 4 show the distribution of MD and PSD of migraine group, according to disease duration. Contrast sensitivity scores did not show statistically significant correlation with the duration of migraine at any spatial frequency $(P>0.05)$.

\section{Discussion}

Migraine is a common neurological condition affecting $10-15 \%$ of adults..$^{11}$ As the visual pathways are involved in the pathophysiology of migraine, it is not surprising that many investigators studied the visual field and contrast processing in involved patients. Visual field deficits have been identified in $30-50 \%$ of migraineurs between migraine episodes. ${ }^{1-5}$ This abnormal function does not seem to result from the direct effect of visual aura on cortical visual pathways, because deficits have been reported in subjects who do not experience visual aura as well as in those who do. ${ }^{1-8}$ In addition to as well as homonymous defects, which shows cortical involvement, ${ }^{12-14}$ unilateral nonhomonymous defects were also detected resembling a more localized defect. ${ }^{1-3,5}$

There is substantial evidence that visual information is transmitted from the retina to cortical area V1 by three pathways that are largely separate, both anatomically
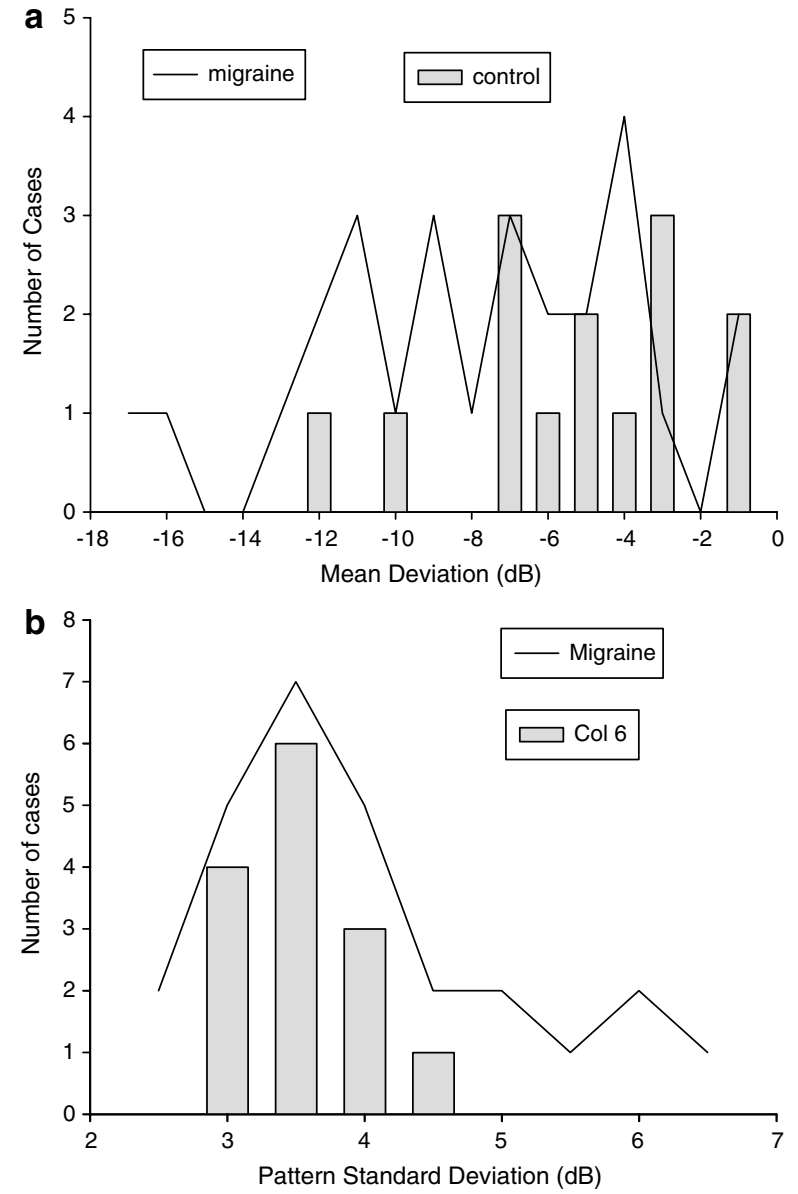

Figure 1 (a) Histogram of the mean deviation (MD) of migraine patients and control subjects. (b) Histogram of the pattern standard deviation (PSD) of migraine patients and control subjects.

Table 3 Spatial-contrast sensitivity scores (F.A.C.T. $^{\text {TM}}$ ) of migraine and control groups

\begin{tabular}{lccc}
\hline $\begin{array}{l}\text { Spatial-contrast } \\
\text { sensitivity }\end{array}$ & Control & Migraine & $\mathrm{P}^{\mathrm{a}}$ \\
\hline $1.5 \mathrm{cpd}$ & $54.5 \pm 15.79$ & $34.02 \pm 13.06$ & $<0.0001^{*}$ \\
$3 \mathrm{cpd}$ & $96.64 \pm 17.70$ & $56.2 \pm 16.2$ & $<0.0001^{*}$ \\
$6 \mathrm{cpd}$ & $111.85 \pm 27.41$ & $60.7 \pm 24.3$ & $<0.0001^{*}$ \\
$12 \mathrm{cpd}$ & $62.96 \pm 22.13$ & $26.9 \pm 11.14$ & $<0.0001^{*}$ \\
$18 \mathrm{cpd}$ & $27.64 \pm 7.76$ & $9.05 \pm 3.18$ & $<0.0001^{*}$ \\
\hline
\end{tabular}

${ }^{*} P<0.05$ indicates statistically significance.

${ }^{a}$ Mann-Whitney $U$ Test.

and functionally. These are parvocellular (P), magnocellular (M), and koniocellular (K) pathways.

Relative to P cells (approximately $80 \%$ of ganglion cells), $\mathrm{M}$ and $\mathrm{K}$ cells are large and sparse. ${ }^{15,16}$ In certain diseases, including glaucoma, ${ }^{17,18}$ visual dysfunction is detected more readily by tests designed to assess the function of $\mathrm{M}$ or K pathways than by those that assess the 
Table 4 Spearman correlations between visual field indices and CS scores in the migraine group

\begin{tabular}{lccrrr}
\hline & \multicolumn{2}{c}{$M D$} & & \multicolumn{2}{c}{$P S D$} \\
\cline { 2 - 3 } \cline { 5 - 6 } Spatial frequency & $\mathrm{r}$ & $\mathrm{P}$ & & $\mathrm{r}$ & $\mathrm{P}^{\mathrm{a}}$ \\
\hline $1.5 \mathrm{cpd}$ & 0.39 & 0.01 & & -0.45 & $0.003^{*}$ \\
$3 \mathrm{cpd}$ & 0.43 & 0.005 & & -0.45 & $0.003^{*}$ \\
$6 \mathrm{cpd}$ & 0.56 & 0.0001 & & -0.51 & $0.001^{*}$ \\
$12 \mathrm{cpd}$ & 0.45 & 0.003 & & -0.53 & $0.0001^{*}$ \\
$18 \mathrm{cpd}$ & 0.48 & 0.0001 & & -0.67 & $0.0001^{*}$ \\
\hline
\end{tabular}

${ }^{*} P<0.05$ indicates statistically significance.

a Mann-Whitney $U$ Test.

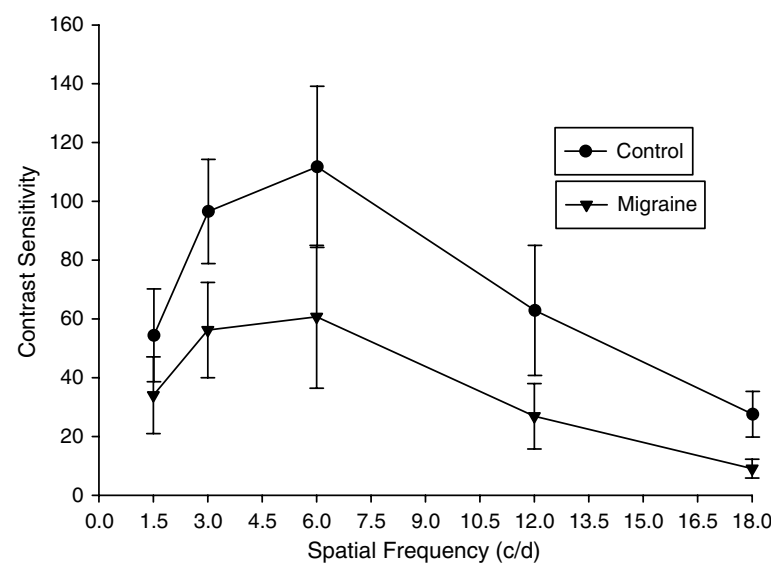

Figure 2 Spatial-contrast sensitivity functions of migraine patients and control subjects. Values are shown as mean \pm SEM.

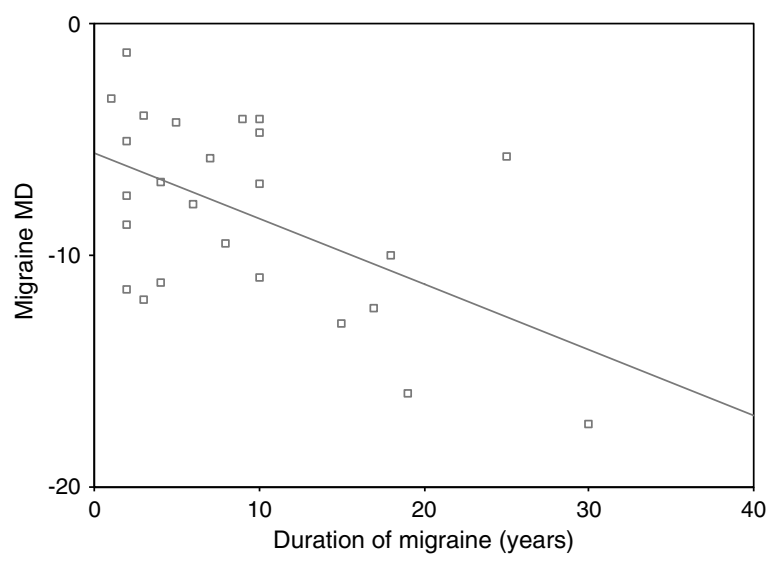

Figure 3 Scattergram showing the correlation between MD and duration of migraine.

function of P pathways. Migraineurs' visual field loss is of no exception. SWAP utilizes a blue stimulus in order to stimulate the blue cones preferentially and a highluminance yellow background to adapt the green and red

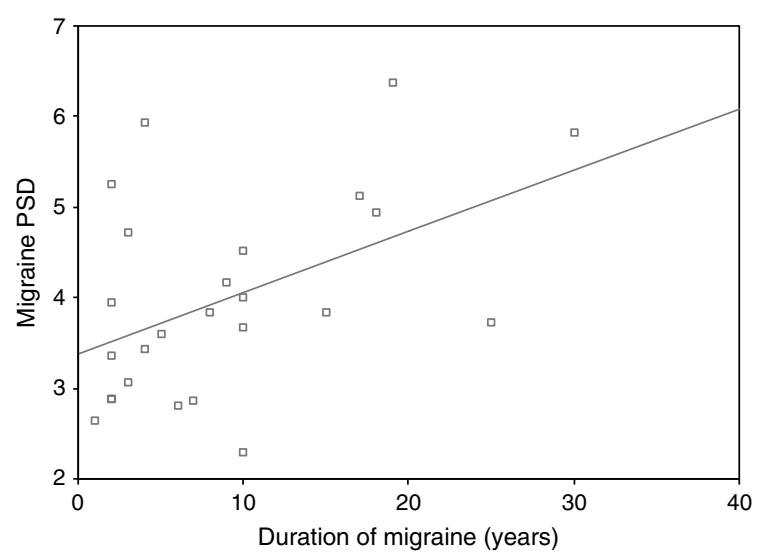

Figure 4 Scattergram showing the correlation between PSD and duration of migraine.

cones and to saturate, simultaneously, the activity of rods. In such a manner, it is designed to test magnocellular pathways. In our study, we found SWAP indices to be significantly higher in migraine subjects than that of controls. This was not a new finding in terms of visual field testing as the same observation was reported by Mc Kendrick et al. ${ }^{5}$ The short-wavelength sensitivity pathways are particularly vulnerable to retinal disease, with evidence of dysfunction of the photoreceptors and postreceptor sites. Diabetes and glaucoma are two ocular diseases particularly involving ganglion cells. As our study population had no diabetes, defects observed here may be more likely to be related with a disease of optic nerve origin like glaucoma.

Although this may look like a direct and weak conclusion from only this study, migraine has been linked to normal tension glaucoma since the report of Phelps and Corbett in 1985. ${ }^{19}$ Neurological evaluation of patients with lowpressure glaucoma in that study revealed a history of common or classic migraine headache in 12 out of 27 patients. The authors later conducted a case-control study to asses whether migraine was really more frequent in patients with low-pressure glaucoma than that in normal subjects, and found that subjects aged 70 years or older had increased prevalence of headache in the low-pressure glaucoma group when compared with the normal group that was marginally statistically significant. ${ }^{20}$ They hypothesized that the increased prevalence of migraine in low-tension glaucoma patients may be due to migraine-related ischaemia. After that, many studies are present in the literature indicating a relationship between glaucoma and migraine either as a cause or a factor for progression. ${ }^{1,2,19-27}$ Recently, visual field abnormalities of migraine patients in SWAP testing was further analysed by us and we found that $53.3 \%$ of migraine patients had abnormal glaucoma hemifield tests, which is highly specific to glaucoma disease. ${ }^{28}$ 
However, although theories may relate the pathophysiology of migraine and normal-tension variant of glaucoma in vascular basis, another recent article by Shephard showed that migarine patients had selective deficits for S-cone discriminations and abnormalities in SWAP testing can be explained with this. ${ }^{29}$

Contrast-sensitivity deficits in migraineurs had been reported previously. ${ }^{9}$ Khalil and Legg $^{9}$ reported decrease in contrast sensitivity with a history of migraine with aura with 30 years or more but normal performance in cases with 10 years or less. Our cases had a disease duration of 30 years or less. We also found that contrast sensitivity defects were correlated with migraine duration, thus decrease in contrast sensitivity may relate to migraine chronicity, which supports Khalil's ${ }^{9}$ findings, but according to our study, not as much as 30 years may be necessary for a deficit to be observed in contrast function.

The second point about contrast sensitivity is that, if a defect present in migraineurs, is it involving either magnocellular or parvacellular pathways? In 2001, a study reported that contrast function is affected in migraine cases after 7 days from an attack at especially lower spatial frequencies indicating a magnocellular defect. ${ }^{15}$ Same investigators later in 2003 conducted a more complex study, which evaluated the $\mathrm{M}$ and $\mathrm{P}$ pathways with steady-pedestal and pulsed-pedestal stimuli corresponding for seven pedestal luminances between 15 and $60 \mathrm{~cd} / \mathrm{m}^{2}$, and found that contrast processing is abnormal in migraine cases at times between episodes in magnocellular pathways in migraine cases with and without aura. ${ }^{4}$ In our study, we also found that at all spatial frequencies, migraineurs had significantly lower contrast, sensitivities than normal controls which is also supported with several other studies. ${ }^{6-8}$

The pathophysiological mechanism underlying contrast-processing dysfunction is unknown. Current evidence supports a neural basis for the aura component of headache known as cortical spreading dysfunction (CSD). CSD result in a decrease in regional cerebral blood flow that spreads from the occipital cortex. ${ }^{30,31}$ Such deficits may represent temporary cell malfunctioning. The significance of these deficits to long-term ocular health in these individuals is currently unknown, but as we already mentioned before data resembling, a relationship between normal tension glaucoma and migraine either as a cause or a factor involved in disease progression is still evolving. ${ }^{25-28,30-32}$ Beyond these, Harle and Evans ${ }^{33}$ suggest an alternative explanation that migraine headache might cause a magnocellular-specific dysfunction unrelated to glaucoma and they claim that they are currently comparing visual fields, ocular tensions, and optic nerve head analysis in migraine and control subjects to investigate this hypothesis. ${ }^{33}$
The findings of this study correlate with several other previous studies, which assessed precortical pathways by vision-related tests in the migraineurs population, which were found to be abnormal. However, we here additionally studied the visual field and contrast sensitivity tasks on same population, which eliminates the ideas of confounding factors (as both tests were done on the same person) of several different studies affecting the results of each tests. Any confounding factor in one test should show itself on the other, but instead we found both of the vision-related tests to be abnormal. Additionally, defects observed in each test correlated significantly with each other. The only point remaining to us is that the migraine characteristics (severity of headache, frequency of attacks, etc.) might have an influence on our results, but we actually did not get any information about this point and after this study, we may design a new one, which will also include a migraine questionaire. So not simply saying that every migraine patient, but only the ones with some disease characteristics (like severe cases with frequent attacks) may have a relation with an eye disease like glaucoma.

The possibility of large number of migraine patients demonstrating abnormal results with visual tasks may raise important questions. First, defects observed in SWAP were of nonselective in nature but presented an evident visual dysfunction in migraine cases. Thus, migraine patients should be excluded from normative databases of visual function. Second, a challenge remains in determining a relationship between glaucoma and migraine. If these deficits in visual-related tests are shown to be progressive and persistent and relate with glaucomatous neuropathy, a different issue will be discussed in the near future, like the use of antiglaucomatous drugs in migraine patients or migraine medications in normal tension glaucoma patients. As primary open angle glaucoma affects $3 \%$ of the population $^{34}$ older than 40 years and within this glaucoma population, estimates of migraine prevalence is between 17 and $25 \%,{ }^{27}$ within the general population, $2.5-7.5 \%,{ }^{27,35}$ may have visual field defects in association with migraine in the presence of normal optic nerve appearance and normal intraocular pressure. Whether migraine populations have a high risk for glaucoma is still unknown and needs further analyses with prospective and more detailed studies.

\section{References}

1 Lewis RA, Vijayan N, Watson C, Keltner J, Johnson CA. Visual field loss in migraine. Ophthalmol 1989; 96: 321-326.

2 De Natale R, Polimeni D, Narbone MC, Scullica MG, Pelicano M. Visual field defects in migraine patients. In: 
Mills RP (ed). Perimetry Update. Amsterdam: Kugler, 1994, pp 283-284.

3 McKendrick AM, Vingrys AJ, Badcock DR, Heywood JT. Visual field losses in subjects with migraine headaches. Invest Ophthalmol Vis Sci 2000; 41: 1239-1247.

4 McKendrick AM, Vingrys AJ, Badcock DR, Heywood JT. Visual dysfunction between migraine events. Invest Ophthalmol Vis Sci 2001; 42: 626-633.

5 McKendrick AM, Cioffi GA, Johnson CA. Short wavelength sensitivity deficits in patients with migraine. Arch Ophthalmol 2002; 120: 154-161.

6 McColl SL, Wilkinson F. Visual contrast gain control in migraine: measures of visual cortical excitability and inhibition. Cephalalgia 2000; 20: 74-84.

7 Shepard AJ. Visual contrast processing in migraine. Cephalalgia 2000; 10: 865-880.

8 Benedek K, Tajti J, Janaky M, Vecsei L, Benedek G. Spatial contrast sensitivity of migraine patients without aura. Cephalalgia 2002; 22: 142-145.

9 Khalil NM, Legg NJ. Pathophysiology of migraine: a study using VEP and contrast sensitivity. In: Clifford and Rose FC (eds). New advances in headache research. 3rd edn. London: Smith-Gordon, 1989, pp 57-61.

10 International Headache Society (1988). Classification and diagnostic criteria for headache disorders, cranial neuralgias and facial pain. Cephalagia 1988; 8: 7-11.

11 Stewart WF, Shechter A, Rasmussen BK. Migraine prevalence: a review of population based studies. Neurology 1994; 44: 17-23.

12 Ebner R. Visual field examination during transient migrainous visual loss. J Clin Neuro-ophthalmol 1991; 11: 114-117.

13 Bowerman LS. Transient visual field loss secondary to migraine. I Am Optom Assoc 1989; 60: 912-916.

14 Wakakura M, Ichibe Y. Permanent homonymous hemianopsia following migraine. J Clin Neuro-ophthalmol 1992; 12: 198-202

15 McKendrick AM, Badcock DR. Contrast-processing dysfunction in both magnocellular and parvocellular pathways in migraineurs with or without aura. Invest Ophthalmol Vis Sci 2003; 44: 442-448.

16 Perry VH, Oehler R, Cowey A. Retinal ganglion cells that project to the dorsal lateral geniculate nucleus in the macaque monkey. Neuroscience 1984; 12: 1102-1123.

17 Sample PA, Bosworth CF, Weinreb RN. Short-wavelength automated perimetry and motion automated perimetry in patients with glaucoma. Arch Ophthalmol 1997; 115: 1129-1133

18 Johnson CA, Adams AJ, Casson EJ, Brandt JD. Progression of early glaucomatous visual field loss for blue-on-yellow and standard white-on-white automated perimetry. Arch Ophthalmol 1993; 111: 652-656.
19 Phelps CD, Corbett JJ. Migraine and low tension glaucoma: a case control study. Invest Ophthalmol Vis Sci 1985; 26 : 1105-1108.

20 Corbett JJ, Phelps CD, Eslinger P, Montague PR. The neurological evaluation of patient with low-tension glaucoma. Invest Ophthalmol Vis Sci 1985; 26: 1101-1104.

21 Drance SM, Sweeney VP, Morgan RW, Feldman F. Studies of factors involved in the production of low-tension glaucoma. Arch Ophthalmol 1973; 89: 457-465.

22 Klein BE, Klein R, Meuer SM, Goetz LA. Migraine headache and its association with open-angle glaucoma. The Beaver Dam Eye Study. Invest Ophthalmol Vis Sci 1993; 34: 3024-3027.

23 Levene RZ. Low tension glaucoma: a critical review and new material. Surv Ophthalmol 1980; 24: 621-664.

24 Nizankowska MH, Turno-Krecicka A, Misiuk-Hojlo M. Coexistence of migraine and glaucoma like visual field defects. Klin Oczna 1997; 99: 121-126.

25 Orgül S, Flammer J. Headache in normal-tension glaucoma patients. J Glaucoma 1994; 3: 292-295.

26 Usui T, Iwata K, Shirakashi M, Abe H. Prevalence of migraine in low-tension glaucoma and primary open-angle glaucoma in Japanese. Br J Ophthalmol 1991; 75: 224-226.

27 Wang JJ, Mitchell P, Smith W. Is there an association between migraine headache and open angle glaucoma? Findings from the Blue Mountains Eye Study. Ophthalmol 1997; 104: 1714-1719.

28 Yenice O, Temel A, Incili B, Tuncer N. Short-wavelength automated perimetry in patients with migraine. Graefe's Arch Clin Exp Ophthalmol; DOI:10.1007/s00417005-0083-7.

29 Shepherd AJ. Colour vison in migraine: selective deficits for S-cone discriminations. Cephalalgia 2005; 25 412-423.

30 Sanchez del Rio M, Bakker D, Wu O, Agosti R, Mitsikostas DD, Ostergaard L et al. Perfusion weighted imaging during migraine: spontaneous visual aura and headache. Cephalalgia 1999; 19: 701-707.

31 Olesen J, Larsen B, Lauritzen M. Focal hyperemia followed by spreading oligemia and impaired activation of $\mathrm{rCBF}$ in classic migraine. Ann Neurol 1981; 9: 344-352.

32 Flammer J. Psychophysical mechanisms and treatment of vasospastic disorders in normal-tension glaucoma. Bull Soc Belge Ophthalmol 1992; 244: 129-134.

33 Harle DE, Evans BJW. The optometric correlates of migraine. Ophthal Physiol Opt 2004; 24: 369-383.

34 Mitchell P, Smith W, Attebo K, Healey PR. Prevalence of open-angle glaucoma in Australia: the Blue Mountains Eye Study. Ophthalmol 1996; 103: 1661-1669.

35 Pradalier A, Hamard P, Sellem E, Bringer L. Migraine and glaucoma: an epidemiologic survey of French ophthalmologists. Cephalalgia 1998; 18: 74-76. 